\title{
Evolution of a Molecular Shape Resonance along a Stretching Chemical Bond
}

\author{
Felix Brausse ${ }^{*},{ }^{\dagger}$ Florian Bach, Faruk Krečinić $\odot,{ }^{\dagger}$ Marc J. J. Vrakking, and Arnaud Rouzée \\ Max-Born-Institut, 12489 Berlin, Germany
}

(Received 10 March 2020; accepted 28 July 2020; published 14 September 2020)

\begin{abstract}
We report experiments on laser-assisted electron recollisions that result from strong-field ionization of photoexcited $\mathrm{I}_{2}$ molecules in the regime of low-energy electron scattering ( $<25 \mathrm{eV}$ impact energy). By comparing differential scattering cross sections extracted from the angle-resolved photoelectron spectra to differential scattering cross sections from quantum-scattering calculations, we demonstrate that the electron-scattering dynamics is dominated by a shape resonance. When the molecular bond stretches during the evolution of a vibrational wave packet this shape resonance shifts to lower energies, both in experiment and theory. We explain this behavior by the nature of the resonance wave function, which closely resembles an antibonding molecular orbital of $\mathrm{I}_{2}$.
\end{abstract}

DOI: 10.1103/PhysRevLett.125.123001

In strong-field ionization of atoms and molecules by linearly polarized laser light, the oscillating laser electric field can cause a photoelectron to recollide with its parent ion. These so-called laser-assisted electron recollisions (LAERs) [1] play a critical role in shaping the observable photoelectron and high-harmonic emission spectra. For strong-field ionization in slowly varying laser fields (i. e., at wavelengths in the near infrared and longer), LAERs have been rationalized in the framework of the quantitativerescattering $(\mathrm{QRS})$ theory $[2,3]$. This approach predicts that certain cuts through the three-dimensional (3D) photoelectron momentum distribution reflect the differential scattering cross sections (DCSs) of the field-free electron-molecular-ion scattering process. In the regime of high electron impact energies, the atoms of a molecule can be treated as point scatterers, an approximation that is known as the independent-atom model (IAM) [4]. The necessary condition for this approximation to hold is that, for an electron returning with kinetic energy $E_{r}$, the de Broglie wavelength $\lambda=h / \sqrt{2 E_{r} m_{e}}$ (with Planck constant $h$ and electron mass $m_{e}$ ) be small compared to the internuclear distances in the molecule. Using the IAM, internuclear distances were recovered from strong-field experiments on a number of small molecules, such as $\mathrm{N}_{2}$ [5], OCS [6], $\mathrm{C}_{2} \mathrm{H}_{2}[7,8], \mathrm{C}_{2} \mathrm{H}_{4}$ [9], and benzene [10].

At scattering energies that are too low for the assumptions of the IAM to hold, the electron scattering dynamics are sensitive to the valence electron distribution. Under certain circumstances, the potential that is associated with

Published by the American Physical Society under the terms of the Creative Commons Attribution 4.0 International license. Further distribution of this work must maintain attribution to the author(s) and the published article's title, journal citation, and DOI. this valence electron distribution can transiently capture an incoming scattering electron through the delicate interplay between attractive (Coulombic) and repulsive (centrifugal) forces that act on the electron, a phenomenon known as a shape resonance [11]. This trapping leads to a strong, characteristic modulation of the DCS over a narrow range of impact energies. In single-photon photoionization experiments, shape resonances manifest themselves as a pronounced variation in the photoabsorption cross section and the photoelectron angular distributions [12,13], and a number of studies have been published in an attempt to relate the energy position of shape resonances to internuclear distances [14-16]. However, it is still an open question to what extent shape resonances can be used to track ultrafast molecular dynamics. In static experiments, shape resonances have been shown to play a key role in shaping the photoelectron momentum distributions resulting from laser-assisted electron recollisions in $\mathrm{O}_{2}$ and $\mathrm{CO}_{2}$ [17]. Recently, Nguyen et al. [18] have presented a detailed theoretical investigation of how the shape resonance in vibrationally excited $\mathrm{SF}_{6}$ molecules evolves in time when probed with attosecond $\mathrm{x}$-ray pulses. An experimental demonstration of this approach, however, has so far remained a desideratum.

Here, we report experiments on the strong-field ionization of $\mathrm{I}_{2}$ at $1.3 \mu \mathrm{m}$ in which a range of internuclear distances were accessed by preparing a vibrational wave packet in the weakly bound $B$ state, which was probed at specific pump-probe delays. With the help of quantumscattering calculations and an approach very similar to that of Nguyen et al. [18], we assign a pronounced maximum in the yield of backscattered photoelectrons as a function of kinetic energy to a strong molecular shape resonance and investigate the dependence of its properties (i. e., energy position and width) on the I-I internuclear distance. Our calculations show that large shifts in the resonance energy 
occur upon increasing the bond length, indicating that molecular shape resonances are a sensitive probe of small changes in internuclear distances.

In the experiments [19], we used a commercial laser system delivering $30 \mathrm{fs}, 3 \mathrm{~mJ}, 800 \mathrm{~nm}$ laser pulses at $1 \mathrm{kHz}$ repetition rate to pump two commercial optical parametric amplifiers (TOPAS $\mathrm{C}$ from Light Conversion). Each TOPAS was pumped by $1 \mathrm{~mJ}$ of input laser energy per pulse, with one generating the $555 \mathrm{~nm}, 90 \mu \mathrm{J}$ pump pulses and the other generating the $1.3 \mu \mathrm{m}, 150 \mu \mathrm{J}$ probe pulses. The pump wavelength was chosen to optimize excitation to the $\mathrm{B}^{3} \Pi_{0 u}^{+}$state of $\mathrm{I}_{2}[41,42]$ (see Supplemental Material [19]). The pump pulses were attenuated to $4 \mu \mathrm{J}$ to suppress multiphoton transitions into higher-lying excited states. Both beams were focused at the center of a velocity map imaging spectrometer (VMIS) [43] using a $250 \mathrm{~mm}$ focaldistance lens. A telescope in the probe arm was used to ensure a smaller focus size for the probe (ionizing) laser pulse. In the interaction region, the two pulses were crossed with a molecular beam obtained by expanding a mixture of iodine in helium at 10 bar of backing pressure through a pulsed Even-Lavie valve operated at $200 \mathrm{~Hz}$. To increase the vapor pressure of $\mathrm{I}_{2}$, the valve was heated and held at a constant temperature of $45^{\circ} \mathrm{C}$. In all experiments, the pump and probe pulses were copolarized, and the polarization axis was parallel to the plane of the detector of the VMI spectrometer. After ionization by the $1.3 \mu \mathrm{m}$ pulse, the charged fragments (ions or electrons) were projected onto a chevron-paired microchannel plate (MCP) detector followed by a phosphor screen. For photoion measurements [19], the MCP-phosphor assembly was gated to enable detection of charged particles with specific mass-overcharge ratios. The 2D projections of the momentum distribution on the phosphor screen were recorded with a CCD camera. From these projections, 3D momentum distributions were reconstructed using the maximumentropy velocity-map image reconstruction method of Dick [44].

Figure 1(a) shows the central slice through the reconstructed photoelectron momentum distribution recorded for the strong-field ionization of $\mathrm{I}_{2}$ in its ground state. To capture the weak rescattering signal with sufficient statistics, the photoelectron signal was accumulated over $3 \times 10^{5}$ laser shots. The concentric circles in Fig. 1(a) divide the angle-resolved photoelectron momentum distribution into three regions. The first region is confined to photoelectrons that have a maximum final kinetic energy $E_{f}$ of twice the ponderomotive energy, $U_{p}=I / 4 \omega^{2}$ (in atomic units), which depends on both the laser intensity $I$ and the laser frequency $\omega$. This upper energy bound for direct photoemission, derived from a purely classical picture of abovethreshold ionization [45], is shown as the black inner dotted circle in Fig. 1(a). However, both the wave nature of the photoelectron wave packet and the finite initial momentum of the outgoing electron soften this limit. As the yield of

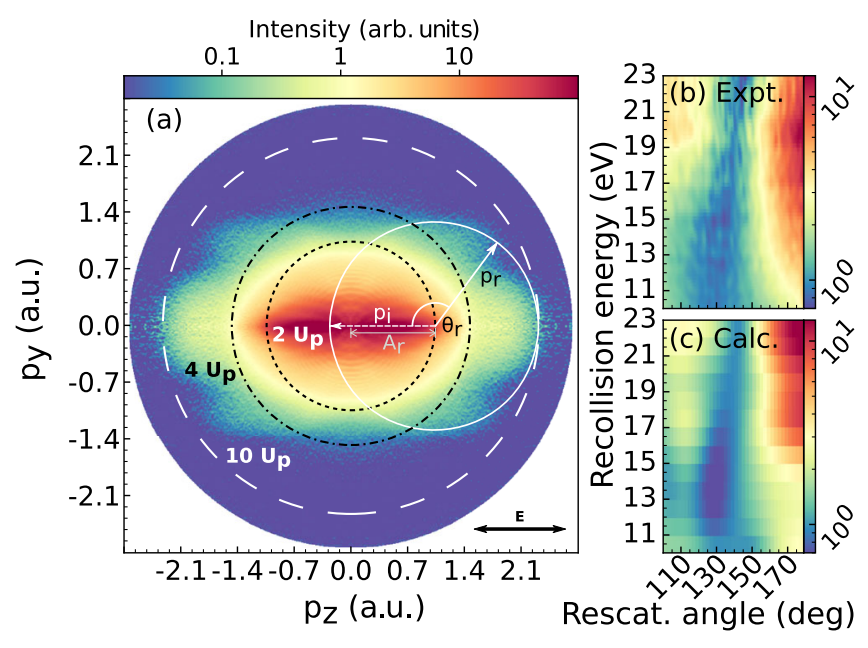

FIG. 1. Extraction of the electron-scattering distributions from a static experiment on ground-state $\mathrm{I}_{2}$ and comparison to calculations invoking the QRS approach. (a) Slice through the inverted 3D momentum distribution, recorded following strongfield ionization of $\mathrm{I}_{2}$ by a $1.3 \mu \mathrm{m}$ pulse with a peak intensity of $4.6 \times 10^{13} \mathrm{~W} / \mathrm{cm}^{2}$. The concentric circles indicate electron kinetic energies of $2 U_{p}, 4 U_{p}$, and $10 U_{p}$, respectively. The solid white circle represents electrons with incoming momentum $p_{i}$ that have elastically scattered into the scattering angle $\theta_{r}$, and are shifted in momentum space by the instantaneous vector potential $-A\left(t_{r}\right)$. (b) DCSs, normalized at a scattering angle of $\theta_{0}=(135 \pm 4)^{\circ}$, extracted from the data in (a). (c) Corresponding ePolyscat QRS calculation of the strong-field ionization of $I_{2}$ in its ground-state and equilibrium geometry.

rescattered electrons is roughly 2 orders of magnitude weaker than that of direct electrons, we restrict our analysis of the former to electrons that have a kinetic energy of at least $4 U_{p}$ (black dash-dotted line). Rescattering produces electrons with kinetic energies of up to $10 U_{p}$ (region 3, dashed line). In other words, we assume that all photoelectrons in the energy range between $4 U_{p}$ and $10 U_{p}$ - the so-called recollision plateau-have undergone rescattering.

To analyze and interpret this recollision plateau, we consider how it forms in a purely classical picture. According to Spanner et al. [46], electrons that rescatter at the time of recollision $t_{r}$ with recollision momentum $p_{r}$ end up on a circle in momentum space that is shifted away from the origin by the instantaneous vector potential at the time of recollision, $-A\left(t_{r}\right)$ [see solid white circle in Fig. 1(a)]. For every ionization time $t_{i}$ within one laser period, solving Newton's equation for motion of an electron in the laser field yields the relation between the recollision time $t_{r}$ and the corresponding recollision momentum $p_{r}\left(t_{r}\right)$. Hence, we can construct a mapping between the experimentally measured momentum distributions and the intensity distribution of the rescattered electrons. This mapping forms the basis of the quantitative rescattering theory $[2,3]$. Also, QRS gives an expression for the angular distribution of the backscattered electrons, 
$D\left(E_{r}, \theta_{r}\right)$, which depends on the recollision energy $E_{r}$ and the rescattering angle $\theta_{r}$. This expression is derived from the differential cross section for a molecule that is fixed in space, $\sigma\left[E_{r}, \boldsymbol{\Omega}=\left(\theta_{r}, \phi_{r}\right), \boldsymbol{\Theta}\right]$, which depends on the molecule's orientation relative to the (fixed) direction of incoming electron, parametrized by the set of Euler angles $\boldsymbol{\Theta}=(\alpha, \beta, \gamma)$. The fixed-in-space cross section is then integrated over all molecular orientations, weighted by the strong-field ionization rate $P_{\text {ion }}(\boldsymbol{\Theta})$ and the molecularaxis distribution of the ensemble $P_{\mathrm{MA}}(\boldsymbol{\Theta})$, i.e.,

$$
\begin{aligned}
D\left(E_{r}, \theta_{r}\right)= & w\left(p_{r}\right) \int_{0}^{\pi} \int_{0}^{2 \pi} P_{\mathrm{ion}}(\boldsymbol{\Theta}) P_{\mathrm{MA}}(\boldsymbol{\Theta}) \\
& \times \sigma\left(E_{r}, \boldsymbol{\Omega}, \boldsymbol{\Theta}\right) d \alpha \sin \beta d \beta .
\end{aligned}
$$

The quantity $w\left(p_{r}\right)$ in Eq. (1) is the momentum distribution of the returning electron wave packet. In writing Eq. (1), we assume that the imprint of the molecular orbital is washed out during propagation, which is why $w\left(p_{r}\right)$ can be factored out of the integral. Also, although $\sigma\left(E_{r}, \boldsymbol{\Omega}, \boldsymbol{\Theta}\right)$ explicitly depends on the azimuthal angle $\phi_{r}$, this dependence is averaged out as both $P_{\text {ion }}(\boldsymbol{\Theta})$ and $P_{\mathrm{MA}}$ are cylindrically symmetric with respect to the laser polarization axis. Moreover, the effect of $w\left(p_{r}\right)$ can be eliminated altogether by normalizing $D\left(E_{r}, \theta_{r}\right)$ at the angle $\theta_{0}$ for which the differential scattering cross sections vary the least as a function $E_{r}$ [17]. When applied to both the measured rescattered electrons and DCSs calculated with Eq. (1), the experiment and theory can be compared to each other.

To model the DCSs (and to determine $\theta_{0}$ ) we carried out quantum-scattering calculations with the ePolyScat software suite developed by Lucchese and co-workers $[47,48]$. In epolyscat, the electron-molecule interaction is treated at the nonrelativistic Hartree-Fock level of theory. To construct the scattering potential, a restricted open-shell HartreeFock self-consistent field calculation was performed for the singly charged molecular iodine $\mathrm{I}_{2}{ }^{+}$in the equilibrium geometry of the neutral molecule with the GAMESS (US) [49] package, using the Sapporo augmented triple- $\zeta$ basis set [50] to obtain the molecular wave function (assuming $C_{\infty v v}$ symmetry to account for symmetry breaking as the bond length is varied). From this single-determinant wave function the full exact static exchange plus model correlation polarization potential was constructed and the corresponding electron-ion scattering wave functions were calculated on an angular grid with spherical harmonics of angular momentum up to $l_{\max }=180$ and an adaptive radial grid [19]. All electron-scattering calculations were carried out for collision energies between 9 and $24 \mathrm{eV}$ (approximately the energy range accessible in the experiment; see below) in steps of $1 \mathrm{eV}$. The angle-dependent standard deviation of the DCSs over this energy range was computed, from which we extracted $\theta_{0}=135^{\circ}$. We note that in the computational model the molecular-orbital strong-field approximation [51] was used to calculate $P_{\text {ion }}(\boldsymbol{\Theta})$ [19],

while $P_{\mathrm{MA}}(\boldsymbol{\Theta})$ was assumed to be uniform. Mapping and normalizing the data of Fig. 1(a) according to the QRS model, we obtain the DCSs shown in Fig. 1(b). The presented data are confined to angles and recollision energies for which the QRS approximation is expected to hold (i.e., $E_{f}>4 U_{p}$ ). At the intensity used in the measurement, $4.6 \times 10^{13} \mathrm{~W} / \mathrm{cm}^{2}$, the electrons acquire only moderate recollision energies, $E_{r}=p_{r}^{2} / 2$, up to $23 \mathrm{eV}$. In the extracted DCSs, we observe a strong backscattering emission (near $180^{\circ}$ ) and a minimum at a scattering angle of $140^{\circ}$. We find a very good agreement between the calculated DCSs [Fig. 1(c)] and the experimental results. In particular, the strong backscattering emission observed experimentally is very well reproduced at all recollision energies, as is the minimum observed at $140^{\circ}$, a well-known feature in electron collisions involving iodine atoms [19].

To examine the bond-length dependence of the rescattering signal, photoelectron momentum distributions were recorded in vibrationally excited $\mathrm{I}_{2}$ molecules. The vibrational wave packet was first characterized by Coulomb explosion imaging [52,53], recording $I^{+}$ion momentum distributions as a function of pump-probe delay [19]. At this pump wavelength, the $B$-state vibrational wave packet has a half revival at a pump-probe delay of $15.6 \mathrm{ps}$. Near the half revival, the wave packet becomes highly localized in the internuclear-distance coordinate and oscillates between inner $\left(R_{\text {in }}=2.78 \AA\right)$ and outer $\left(R_{\text {out }}=3.68 \AA\right)$ turning points defined by the $B$-state potential energy curve [54]. To extract the time-dependent changes in the measured DCSs, the data taken at the inner turning point were subtracted from those taken at the outer one, as shown in Fig. 2(a). In this map, a positive value means a larger contribution at the outer turning point than at the inner turning point while a negative value (blue) indicates a larger contribution at the inner turning point. The maximum

(a) Experiment

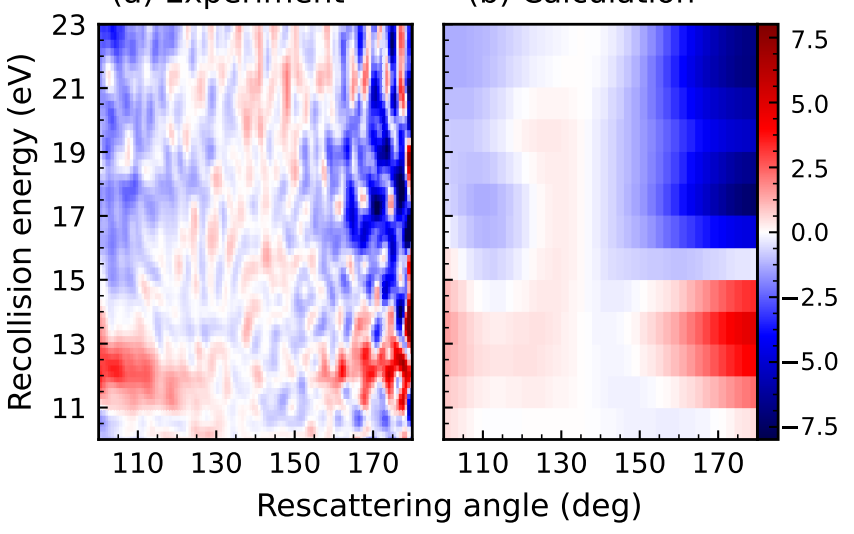

FIG. 2. Absolute difference between DCSs measured (a) and calculated (b) at the outer $\left(R_{\text {out }}=3.68 \AA\right)$ and inner turning points $\left(R_{\mathrm{in}}=2.78 \AA\right)$ for the case of an $\mathrm{I}_{2}$ vibrational wave packet created by $555 \mathrm{~nm}$ wavelength excitation of the $B$ state. 
difference is observed in the backscattering direction $\left(180^{\circ}\right)$, with a strong negative contribution observed at $18 \mathrm{eV}$ and a positive one at $13 \mathrm{eV}$. This observation is perfectly reproduced by the epolyscat QRS model, Fig. 2(b), if we subtract a calculation using $R_{\text {in }}$ from one using $R_{\text {out }}$.

We hypothesize that the pronounced backscattering features can be attributed to shape resonances, following Okunishi et al. [17] in their interpretation of static experiments in $\mathrm{O}_{2}$ and $\mathrm{CO}_{2}$. To verify the presence of a shape resonance in the calculation, we investigate the energy dependence of the electron-ion scattering phase shifts, in which a shape resonance is expected to manifest as a narrow, positive phase jump of $\approx \pi$ in one of the scattering channels [11]. The total scattering wave function can be decomposed into a set of scattering channels, each of which transforms like one of the irreducible representations of the molecular point group [55]. Because of the rapid change of the scattering phase, the cross section-which is related to the first derivative of the scattering phase-is also expected to vary rapidly. In the present case, a sudden jump in the phase can be clearly identified, see Fig. 3(a), for $\mathrm{I}_{2}{ }^{+}$at the equilibrium bond length of the neutral species around an electron kinetic energy of $18 \mathrm{eV}$ for the $E_{1}$ channel (which comprises all partial waves with angular momentum $l \geq 1$ and magnetic quantum number $m= \pm 1$ ). At the same time, this is the impact energy for which a large increase of backscattering is observed in Figs. 1(b) and 1(c). We therefore assign this feature to the shape resonance.

To characterize the shape resonance further, we invoke its formal definition, according to which a shape resonance corresponds to a pole in the complex-energy plane of the scattering $\mathbf{T}$ matrix at the complex-valued energy $\epsilon-i \Gamma / 2$ [11], determining the resonance energy $\epsilon$ and its width $\Gamma$. The advantage of this definition is that the scattering wave function can be evaluated at the pole position, which yields the pure resonance wave function, isolated from other scattering contributions. To identify the poles of the $\mathbf{T}$ matrix we used the pole-searching algorithm of Stratmann and Lucchese [16] that is implemented in epolyscat. In this algorithm, only a simplified scattering potential is used, in which the exact treatment of electron exchange is replaced by a free-electron-gas model exchange, but we find that this simplification leaves the overall scattering dynamics unaltered. The pole-searching calculation identified a pole at $\epsilon \approx 18.0 \mathrm{eV}$. The corresponding scattering wave function is shown as an inset of Fig. 3(b). Around the two nuclei, the resonance wave function closely resembles an antibonding combination of two atomic $f$ orbitals (but note that, different from a molecular orbital, the resonance wave function does not vanish at infinity). Correspondingly, it stands to reason that the degree of destructive interference between the two atomic orbitals and therefore the energy position of the shape resonance should depend on the internuclear distance. Repeating the pole search for (a)

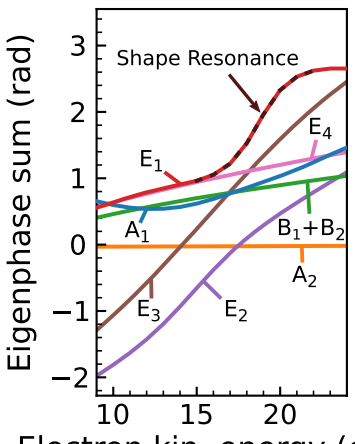

(b)

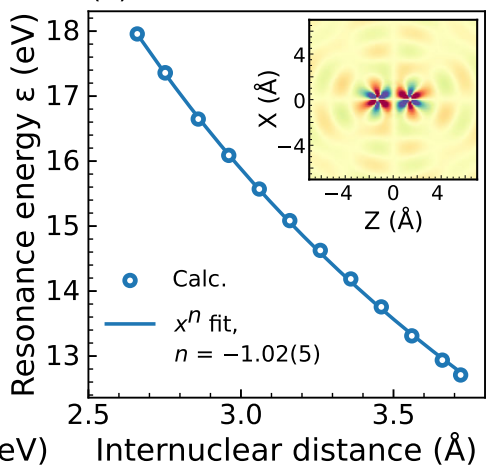

FIG. 3. Identification and bond-length dependence of the molecular shape resonance in $I_{2}$. (a) Calculated evolution of the eigenphase sums as a function of the electron kinetic energy for $\mathrm{I}_{2}{ }^{+}$at the equilibrium internuclear distance of neutral $\mathrm{I}_{2}$. The symbols denote the symmetries (irreducible representations) of all open scattering channels that contribute to the overall scattering wave function, assuming $C_{10}$ v symmetry for $\mathrm{I}_{2}{ }^{+}$ [19]. The dashed line on the curve for the $E_{1}$ channel highlights the characteristic phase jump that is caused by the shape resonance. (b) Energy positions $\epsilon$ of the dominant pole in the complex-energy plane of the scattering $\mathbf{T}$ matrix obtained from the pole-searching algorithm (circles) for various internuclear distances, together with a power-law fit (solid line). The inset shows the real part of the scattering wave function at the equilibrium internuclear distance. The atom positions are located exactly in the centers of the rosette-shaped features.

internuclear distances between 2.66 and $3.86 \AA$, we find that the resonance energy $\epsilon$ is shifted down by $5 \mathrm{eV}$ when the bond length is extended by roughly $0.7 \AA$, scaling approximately with $1 / R$ [see Fig. 3(b)]. As a consequence, the results of Fig. 2 are fully explained by assigning the negative values in the differential DCSs at $18 \mathrm{eV}$ and the positive values at $13 \mathrm{eV}$ to a shift of the shape resonance as the molecule moves toward larger internuclear distances. The $5 \mathrm{eV}$ difference between these two extrema closely matches the shift in resonance energy $\Delta \epsilon$ predicted by the pole-searching calculations.

As the large-angle backscattering is the key factor toward understanding the bond-length dependence of the rescattering process, we have extracted its time dependence by comparing the time-dependent difference signal to that at the delay of the inner turning point, $t_{\mathrm{in}}$,

$$
\Delta D\left(E_{r}, \theta_{r}, t\right)=D\left(E_{r}, \theta_{r}, t\right)-D\left(E_{r}, \theta_{r}, t_{\text {in }}\right),
$$

restricting the analysis to scattering angles between $160^{\circ}$ and $180^{\circ}$, as shown in Fig. 4(a). When the wave packet reaches the outer turning point, the differential features in the experiment at 13 and $18 \mathrm{eV}$ reach their greatest amplitude, as well. This is also true for the epolyscat QRS calculations, Fig. 4(b), which were treated in the same way as the experimental data, while applying a $90 \mathrm{fs}$ temporal convolution to account for the limited time 


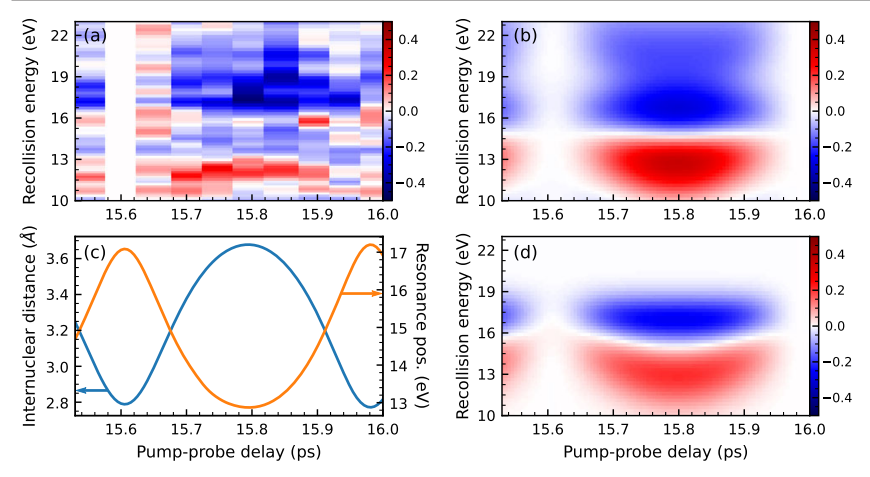

FIG. 4. Time-dependent evolution of the large-angle backscattering signal, shown as the absolute difference to the results obtained for a pump-probe delay of $t=15.6 \mathrm{ps,} \mathrm{which} \mathrm{corre-}$ sponds to the inner turning point [cf. Eq. (2)], for (a) the experiment, (b) the epolyscat QRS calculations, and (d) the simple model. The modeled results, (b) and (d), rely on knowledge of $R(t)$ [blue line in (c)], which was extracted from the photoion imaging results [19]. For the simple model (d), we approximate the isolated shape-resonance contribution by a Gaussian function. The time-dependent energy [orange line in (c)] and width [19] of the Gaussian function as a function of the internuclear distance $R(t)$ were obtained according to the results of the pole-searching calculations [Fig. 3(b)].

resolution in the experiment. To put our interpretation to the test that the shape-resonance detuning is responsible for this observation, we have moreover constructed a very simple model. We approximate the backscattering signal as a function of time delay through a Gaussian centered at the shape-resonance energy position $\epsilon[R(t)]$ [see Fig. 4(c)] with width $\Gamma[R(t)]$, obtained from the pole-searching calculations [19]. Applying the same temporal convolution and subtraction [Eq. (2)] that were used for the full calculations to this simple model, we find that it reproduces the main observations of both the experiment and the full calculations [Fig. 4(d)], in strong support of our interpretation of the experimental and numerical results in terms of shaperesonance dynamics.

In conclusion, laser-assisted low-energy electron recollisions in vibrationally excited $\mathrm{I}_{2}$ molecules were investigated both experimentally and theoretically. Two strong backscattering peaks were observed, namely one that vanishes with increasing bond length and one that appears, centered at a lower scattering energy. Our theoretical investigation shows that the observed dynamics can be explained by the shift in energy of the shape resonance that occurs as the molecule undergoes vibrational motion. Our results prove that low-energy electron-molecular-ion scattering is very sensitive to the dynamics of shape resonances, creating new opportunities for investigating photoinduced molecular dynamics with high temporal and spatial resolution.

M. J. J. V. and A. R. would like to thank the Deutsche Forschungsgemeinschaft, via Project VR 76/1-1 and
Schwerpunktprogramm 1840 (SPP 1840 QUTIF project) via Project RO 4577/4-2, respectively, for support. This project has benefitted from funding from the European Union's Horizon 2020 research and innovation programme under the Marie Sklodowska-Curie Grant Agreement No. 674960 (ASPIRE). We would like to acknowledge Jochen Mikosch and Frank Noack for their support in setting up the experiment.

*brausse@lbl.gov

†Present address: Chemical Sciences Division, Lawrence Berkeley National Laboratory, Berkeley, California 94720, USA.

†Present address: Fritz-Haber-Institut der Max-PlanckGesellschaft, 14195 Berlin, Germany.

[1] C. J. Joachain, N. J. Kylstra, and R. M. Potvliege, Atoms in Intense Laser Fields (Cambridge University Press, Cambridge, England, 2011).

[2] Z. Chen, A.-T. Le, T. Morishita, and C. D. Lin, Phys. Rev. A 79, 033409 (2009).

[3] J. Xu, Z. Chen, A.-T. Le, and C. D. Lin, Phys. Rev. A 82, 033403 (2010).

[4] R. Srinivasan, V.A. Lobastov, C.-Y. Ruan, and A. H. Zewail, Helv. Chim. Acta 86, 1761 (2003).

[5] C. I. Blaga, J. Xu, A. D. DiChiara, E. Sistrunk, K. Zhang, P. Agostini, T. A. Miller, L. F. DiMauro, and C. D. Lin, Nature (London) 483, 194 (2012).

[6] E. T. Karamatskos, G. Goldsztejn, S. Raabe, P. Stammer, T. Mullins, A. Trabattoni, R. R. Johansen, H. Stapelfeldt, S. Trippel, M. J. J. Vrakking, J. Küpper, and A. Rouzée, J. Chem. Phys. 150, 244301 (2019).

[7] M. G. Pullen, B. Wolter, A.-T. Le, M. Baudisch, M. Hemmer, A. Senftleben, C. D. Schroeter, J. Ullrich, R. Moshammer, C. D. Lin, and J. Biegert, Nat. Commun. 6 (2015).

[8] B. Wolter, M. G. Pullen, A.-T. Le, M. Baudisch, K. Doblhoff-Dier, A. Senftleben, M. Hemmer, C. D. Schroeter, J. Ullrich, T. Pfeifer, R. Moshammer, S. Graefe, O. Vendrell, C. D. Lin, and J. Biegert, Science 354, 308 (2016).

[9] Y. Ito, R. Carranza, M. Okunishi, R. R. Lucchese, and K. Ueda, Phys. Rev. A 96, 053414 (2017).

[10] Y. Ito, C. Wang, A.-T. Le, M. Okunishi, D. Ding, C. D. Lin, and K. Ueda, Struct. Dyn. 3, 034303 (2016).

[11] J. Taylor, Scattering Theory: The Quantum Theory of Nonrelativistic Collisions, Dover Books on Engineering (Dover Publications, New York, 2012).

[12] J. L. Dehmer, D. Dill, and S. Wallace, Phys. Rev. Lett. 43, 1005 (1979).

[13] N. Watanabe, J. Adachi, K. Soejima, E. Shigemasa, A. Yagishita, N. G. Fominykh, and A. A. Pavlychev, Phys. Rev. Lett. 78, 4910 (1997).

[14] J. Stöhr, NEXAFS Spectroscopy, in Springer Series in Surface Sciences Vol. 25 (Springer-Verlag, Berlin, 1992).

[15] M. Piancastelli, J. Electron Spectrosc. Relat. Phenom. 100, 167 (1999).

[16] R. E. Stratmann and R. R. Lucchese, J. Chem. Phys. 97, 6384 (1992). 
[17] M. Okunishi, H. Niikura, R. R. Lucchese, T. Morishita, and K. Ueda, Phys. Rev. Lett. 106, 063001 (2011).

[18] N.-T. Nguyen, R. R. Lucchese, C. D. Lin, and A.-T. Le, Phys. Rev. A 93, 063419 (2016).

[19] See Supplemental Material at http://link.aps.org/ supplemental/10.1103/PhysRevLett.125.123001 for a scheme of the experimental setup (Fig. S1), a synopsis of the relevant potential energy curves (Fig. S2), results of the Coulomb-explosion imaging experiments, and details about all calculations presented here (quantum scattering calcs., molecular-orbital strong-field approximation calcs., polesearching calcs.). The Supplementary Material includes Refs. [20-40].

[20] W. A. de Jong, L. Visscher, and W. C. Nieuwpoort, J. Chem. Phys. 107, 9046 (1997).

[21] L. Fang and G. N. Gibson, Phys. Rev. Lett. 100, 103003 (2008).

[22] H. Chen, L. Fang, V. Tagliamonti, and G. N. Gibson, Phys. Rev. A 84, 043427 (2011).

[23] J. W. Cooley, Math. Comput. 15, 363 (1961).

[24] R. J. L. Roy, J. Quant. Spectrosc. Radiat. Transfer 186, 158 (2017).

[25] S. Gerstenkorn and P. Luc, Opt. Commun. 36, 322 (1981).

[26] H. Miyagi, T. Morishita, and S. Watanabe, Phys. Rev. A 85, 022708 (2012).

[27] R. Lucchese and V. McKoy, Phys. Rev. A 28, 1382 (1983).

[28] A. Temkin, K. V. Vasavada, E. S. Chang, and A. Silver, Phys. Rev. 186, 57 (1969).

[29] R. Lucchese and V. McKoy, Phys. Rev. A 24, 770 (1981).

[30] P. G. Burke, N. Chandra, and F. A. Gianturco, J. Phys. B 5, 2212 (1972).

[31] C.-W. Lee, Phys. Rev. A 58, 4581 (1998).

[32] P. Schwerdtfeger and J. K. Nagle, Mol. Phys. 117, 1200 (2019).

[33] N. T. Padial and D. W. Norcross, Phys. Rev. A 29, 1742 (1984).

[34] P. W. Atkins, M. S. Child, and C. S. G. Phillips, Tables for Group Theory, reprint ed. (Oxford University Press, London, 1984).
[35] S. L. Altmann and P. Herzig, Point-Group Theory Tables, 2nd ed. (Oxford University Press, Oxford, 2011).

[36] J. Muth-Böhm, A. Becker, and F. H. M. Faisal, Phys. Rev. Lett. 85, 2280 (2000).

[37] B. Zhang and Z. Zhao, Comput. Phys. Commun. 192, 330 (2015).

[38] H. R. Reiss, Phys. Rev. A 22, 1786 (1980).

[39] M. Grade and W. Rosinger, Surf. Sci. 156, 920 (1985).

[40] A. Kramida, Yu. Ralchenko, and J. Reader (NIST ASD Team), NIST Atomic Spectra Database, ver. 5.7.1 (National Institute of Standards and Technology, Gaithersburg, MD, 2019), https://physics.nist.gov/asd.

[41] J. Tellinghuisen, J. Chem. Phys. 58, 2821 (1973).

[42] J. Tellinghuisen, J. Chem. Phys. 135, 054301 (2011).

[43] A. T. J. B. Eppink and D. H. Parker, Rev. Sci. Instrum. 68, 3477 (1997).

[44] B. Dick, Phys. Chem. Chem. Phys. 16, 570 (2014).

[45] P. B. Corkum, Phys. Rev. Lett. 71, 1994 (1993).

[46] M. Spanner, O. Smirnova, P. B. Corkum, and M. Y. Ivanov, J. Phys. B 37, L243 (2004).

[47] F. A. Gianturco, R. R. Lucchese, and N. Sanna, J. Chem. Phys. 100, 6464 (1994).

[48] A. P. P. Natalense and R. R. Lucchese, J. Chem. Phys. 111, 5344 (1999).

[49] M. W. Schmidt, K. K. Baldridge, J. A. Boatz, S. T. Elbert, M. S. Gordon, J. H. Jensen, S. Koseki, N. Matsunaga, K. A. Nguyen, S. Su, T. L. Windus, M. Dupuis, and J. A. Montgomery, Jr., J. Comput. Chem. 14, 1347 (1993).

[50] T. Koga, S. Yamamoto, T. Shimazaki, and H. Tatewaki, Theor. Chem. Acc. 108, 41 (2002).

[51] B. Zhang and Z. Zhao, Phys. Rev. A 82, 035401 (2010).

[52] H. Stapelfeldt, E. Constant, and P. B. Corkum, Phys. Rev. Lett. 74, 3780 (1995).

[53] H. Stapelfeldt, E. Constant, H. Sakai, and P. B. Corkum, Phys. Rev. A 58, 426 (1998).

[54] I. Fischer, M. Vrakking, D. Villeneuve, and A. Stolow, Chem. Phys. 207, 331 (1996).

[55] J. B. Diamond, Chem. Phys. Lett. 20, 63 (1973). 